\title{
Global Attractivity of a Rational Difference Equation
}

\author{
Nouressadat Touafek ${ }^{1}$ and Yacine Halim ${ }^{2, *}$ \\ ${ }^{1}$ LMAM Laboratory, Department of Mathematics, University of Jijel, Algeria \\ ${ }^{2}$ Institute of Science and Technology, University Center of Mila, Algeria
}

Received: 23 Feb. 2013, Revised: 3 May. 2013, Accepted: 4 May. 2013

Published online: 1 Sep. 2013

Abstract: In this paper, we investigate the global attractivity of positive solutions of the nonlinear difference equation

$$
x_{n+1}=\frac{a x_{n}^{3}+b x_{n} x_{n-1}^{2}+c x_{n}^{2} x_{n-1}+d x_{n-1}^{3}}{A x_{n}^{3}+B x_{n} x_{n-1}^{2}+C x_{n}^{2} x_{n-1}+D x_{n-1}^{3}}, n=0,1, \ldots
$$

where the parameters $a, b, c, d, A, B, C, D$ are positive real numbers and the initial values $x_{0}, x_{-1}$ are arbitrary positive numbers.

Keywords: Difference equations, global attractivity, periodicity.

\section{Introduction and preliminaries}

Difference equations appear naturally as discrete analogues and as numerical solutions of differential and delay differential equations which model phenomena in ecology, economy, automatic control theory and so forth. Recently, there has been a great attention in studying nonlinear difference equations, see, for instance, [1], [3], [5], [10], [11], and references cited therein, as well as in studying systems of difference equations (see, e.g. [6], [9]).

Consider the following second-order difference equation

$$
x_{n+1}=\frac{a x_{n}^{3}+b x_{n} x_{n-1}^{2}+c x_{n}^{2} x_{n-1}+d x_{n-1}^{3}}{A x_{n}^{3}+B x_{n} x_{n-1}^{2}+C x_{n}^{2} x_{n-1}+D x_{n-1}^{3}}, n=0,1, \ldots
$$

where the initial conditions $x_{0}, x_{-1} \in(0, \infty)$ and the parameters $a, b, c, d, A, B, C, D \in(0, \infty)$.

In this paper, we study the stability of the unique positive equilibrium point $\bar{x}=\frac{a+b+c+d}{A+B+C+D}$, the boundedness and the convergence of the solutions of the equation (1).

Now, we review some definitions (see for example [7], [8]), which will be useful in the sequel.

Let $I$ be an interval of real numbers and let $F: I \times I \longrightarrow$ $I$ be a continuously differentiable function. Consider the difference equation

$$
x_{n+1}=F\left(x_{n}, x_{n-1}\right)
$$

with initial values $x_{-1}, x_{0} \in I$.

Definition 11 A point $\bar{x} \in I$ is called an equilibrium point of Eq.(2) if

$$
\bar{x}=F(\bar{x}, \bar{x}) .
$$

Definition 12 Let $\bar{x}$ be an equilibrium point of Eq.(2).

(i) The equilibrium $\bar{x}$ is called locally stable iffor every $\varepsilon>0$, there exist $\delta>0$ such that for all $x_{-1}, x_{0} \in I$ with $\left|x_{-1}-\bar{x}\right|+\left|x_{0}-\bar{x}\right|<\delta$, we have $\left|x_{n}-\bar{x}\right|<\varepsilon$, for all $n \geq$ -1 .

(ii) The equilibrium $\bar{x}$ is called locally asymptotically stable if it is locally stable, and if there exists $\gamma>0$ such that if $x_{-1}, x_{0} \in I$ and $\left|x_{-1}-\bar{x}\right|+\left|x_{0}-\bar{x}\right|<\gamma$ then

$$
\lim _{n \rightarrow+\infty} x_{n}=\bar{x}
$$

(iii) The equilibrium $\bar{x}$ is called global attractor if for all $x_{-1}, x_{0} \in I$, we have

$$
\lim _{n \rightarrow+\infty} x_{n}=\bar{x}
$$

(iv) The equilibrium $\bar{x}$ is called global asymptotically stable if it is locally stable and a global attractor.

\footnotetext{
*Corresponding author e-mail: halyacine@yahoo.fr
} 
(v) The equilibrium $\bar{x}$ is called unstable if it is not stable.

(Vi) Let $p=\frac{\partial F}{\partial x}(\bar{x}, \bar{x})$ and $q=\frac{\partial F}{\partial y}(\bar{x}, \bar{x})$. Then the equation

$$
y_{n+1}=p y_{n}+q y_{n-1}, n=0,1, \ldots
$$

is called the linearized equation of Eq.(2) about the equilibrium point $\bar{x}$.

The next result, which was given by Clark [2], provides a sufficient condition for the locally asymptotically stability of Eq. (3).

Theorem 11 Consider the difference equation (3) with $p$, $q \in \mathbb{R}$. Then,

$$
|p|+|q|<1
$$

is a sufficient condition for the locally asymptotically stability.

Definition 13 The difference equation (2) is said to be permanent, if there exist numbers $\alpha, \beta$ with $0<\alpha \leq \beta<\infty$ such that for any initial $x_{-1}, x_{0} \in$ I there exists a positive integer $N$ wich depends on the initial conditions such that $\alpha \leq x_{n} \leq \beta$ for all $n \geq N$.

\section{Main results}

Let $f:(0,+\infty)^{2} \rightarrow(0,+\infty)$ be the function defined by

$$
f(x, y)=\frac{a x^{3}+b x y^{2}+c x^{2} y+d y^{3}}{A x^{3}+B x y^{2}+C x^{2} y+D y^{3}} .
$$

In the sequel we need the following real numbers:

$r_{1}=a B-b A, r_{2}=a C-c A, r_{3}=a D-d A, r_{4}=c B-b C$, $r_{5}=b D-d B, r_{6}=c D-d C$.

Lemma 21 1. Assume that

- $\frac{a}{A} \geq \max \left(\frac{b}{B}, \frac{c}{C}\right)$,

- $\frac{d}{D} \leq \min \left(\frac{b}{B}, \frac{c}{C}\right)$,

- $3 r_{3}+r_{4} \geq 0$.

Then, $f$ is nondecreasing in $x$ for each $y$ and it is nonincreasing in $y$ for each $x$.

2. Assume that

- $\frac{a}{A} \leq \min \left(\frac{b}{B}, \frac{c}{C}\right)$,

- $\frac{d}{D} \geq \max \left(\frac{b}{B}, \frac{c}{C}\right)$,

- $3 r_{3}+r_{4} \leq 0$.

Then, $f$ is nonincreasing in $x$ for each $y$ and it is nondecreasing in $y$ for each $x$.

Proof.

1. We have, $3 r_{3}+r_{4} \geq 0$ and it is easy to see that $\frac{a}{A} \geq$ $\max \left(\frac{b}{B}, \frac{c}{C}\right)$ and $\frac{d}{D} \leq \max \left(\frac{b}{B}, \frac{c}{C}\right)$ implies $r_{1}, r_{2}, r_{5}, r_{6} \geq$ 0 . So the result follows from the two formulaes

$$
\begin{aligned}
\frac{\partial f}{\partial x}(x, y) & =\frac{r_{2} x^{4} y+2 r_{1} x^{3} y^{2}+\left(3 r_{3}+r_{4}\right) x^{2} y^{3}}{\left(A x^{3}+B x y^{2}+C x^{2} y+D y^{3}\right)^{2}} \\
& +\frac{2 r_{6} x y^{4}+r_{5} y^{5}}{\left(A x^{3}+B x y^{2}+C x^{2} y+D y^{3}\right)^{2}}, \\
\frac{\partial f}{\partial y}(x, y) & =\frac{-r_{2} x^{5}-2 r_{1} x^{4} y-\left(3 r_{3}+r_{4}\right) x^{3} y^{2}}{\left(A x^{3}+B x y^{2}+C x^{2} y+D y^{3}\right)^{2}} \\
& +\frac{-2 r_{6} x^{2} y^{3}-r_{5} x y^{4}}{\left(A x^{3}+B x y^{2}+C x^{2} y+D y^{3}\right)^{2}} .
\end{aligned}
$$

2. The proof of 2) is similar and will be omitted.

Theorem 21 Assume that

$$
\frac{2\left|2 r_{1}+r_{2}+3 r_{3}+r_{4}+r_{5}+2 r_{6}\right|}{(a+b+c+d)(A+B+C+D)}<1 .
$$

Then the positive equilibrium point $\bar{x}=\frac{a+b+c+d}{A+B+C+D}$ of $E q$. (1) is locally asymptotically stable.

Proof. The linearized equation of Eq. (1) about $\bar{x}=\frac{a+b+c+d}{A+B+C+D}$ is

$$
x_{n+1}=p x_{n}+q x_{n-1}
$$

where

$$
\begin{gathered}
p=\frac{\left(2 r_{1}+r_{2}+3 r_{3}+r_{4}+r_{5}+2 r_{6}\right)}{(a+b+c+d)(A+B+C+D)}, \\
q=-\frac{\left(2 r_{1}+r_{2}+3 r_{3}+r_{4}+r_{5}+2 r_{6}\right)}{(a+b+c+d)(A+B+C+D)} .
\end{gathered}
$$

So, by theorem (11) we get that $\bar{x}$ is locally asymptotically stable if

$$
\frac{2\left|2 r_{1}+r_{2}+3 r_{3}+r_{4}+r_{5}+2 r_{6}\right|}{(a+b+c+d)(A+B+C+D)}<1 .
$$

In the following theorem we prove the permanent of the difference equation (1).

Theorem 22 Let $\left\{x_{n}\right\}_{n=-1}^{+\infty}$ be a positive solution of equation (1).

\section{Assume that}

- $\frac{a}{A} \geq \max \left(\frac{b}{B}, \frac{c}{C}\right)$,

- $\frac{d}{D} \leq \min \left(\frac{b}{B}, \frac{c}{C}\right)$,

Then,

$$
\frac{d}{D} \leq x_{n} \leq \frac{a}{A}
$$

for all $n \geq 1$.

2. Assume that

- $\frac{a}{A} \leq \min \left(\frac{b}{B}, \frac{c}{C}\right)$,

- $\frac{d}{D} \geq \max \left(\frac{b}{B}, \frac{c}{C}\right)$, 
Then,

$$
\frac{a}{A} \leq x_{n} \leq \frac{d}{D}
$$

for all $n \geq 1$.

Proof. 1. We have

$$
\begin{aligned}
& x_{n+1}-\frac{a}{A}=\frac{-r_{2} x_{n}^{2} x_{n-1}-r_{1} x_{n} x_{n-1}^{2}-r_{3} x_{n-1}^{3}}{A\left(A x_{n}^{3}+B x_{n} x_{n-1}^{2}+C x_{n}^{2} x_{n-1}+D x_{n-1}^{3}\right)}, \\
& x_{n+1}-\frac{d}{D}=\frac{r_{3} x_{n}^{3}+r_{6} x_{n}^{2} x_{n-1}+r_{5} x_{n} x_{n-1}^{2}}{D\left(A x_{n}^{3}+B x_{n} x_{n-1}^{2}+C x_{n}^{2} x_{n-1}+D x_{n-1}^{3}\right)} .
\end{aligned}
$$

Now using the fact that $r_{1}, r_{2}, r_{3}, r_{5}, r_{6} \geq 0$, it follows that

$$
\frac{d}{D} \leq x_{n} \leq \frac{a}{A}
$$

for all $n \geq 1$.

2. The proof of 2) is similar and will be omitted.

Here we study the global asymptotic stability of Eq.(1).

\section{Theorem 23 Let}

$$
\begin{aligned}
p_{1} & =-D a+A b+(A+C) d, \\
p_{2} & =-(B+D) a+(A+C) b+(A-D) c+(A+B+C) d, \\
p_{3} & =(A-B-C-D) a+(A+B+C-D) b \\
& +(A-B+C-D) c+(A+B+C+D) d .
\end{aligned}
$$

Assume that

- $\frac{a}{A} \geq \max \left(\frac{b}{B}, \frac{c}{C}\right)$,

- $\frac{d}{D} \leq \min \left(\frac{b}{B}, \frac{c}{C}\right)$,

- $3 r_{3}+r_{4} \geq 0$,

- $\frac{2\left(2 r_{1}+r_{2}+3 r_{3}+r_{4}+r_{5}+2 r_{6}\right)}{(a+b+c+d)(A+B+C+D)}<1$,

- $p_{1}, p_{2}, p_{3} \geq 0$.

Then the equilibrium point $\bar{x}=\frac{a+b+c+d}{A+B+C+D}$ of Eq.(1) is globally asymptotically stable.

Proof. Let $\left\{x_{n}\right\}_{n=-1}^{+\infty}$ be a positive solution of equation (1). In view of theorem (21), we need only to prove that $\bar{x}$ is global attractor.

Let

$$
m=\lim _{n \rightarrow+\infty} \inf x_{n}
$$

and

$$
M=\lim _{n \rightarrow+\infty} \operatorname{supx}_{n}
$$

To prove that

$$
\lim _{n \rightarrow+\infty} x_{n}=\bar{x}
$$

it suffices to show that $m=M$.

Let $\varepsilon \in] 0, m\left[\right.$, then there exist $n_{0} \in \mathbb{N}$ such that for all $n \geq$ $n_{0}$ we get

$$
m-\varepsilon \leq x_{n} \leq M+\varepsilon .
$$

Thus by using lemma (21), Part 1 ; we get for all $n \geq n_{0}+1$

$$
x_{n+1} \geq f(m-\varepsilon, M+\varepsilon),
$$

$$
x_{n+1} \leq f(M+\varepsilon, m-\varepsilon) .
$$

Then, we get the following inequalities

$$
\begin{aligned}
& m \geq f(m-\varepsilon, M+\varepsilon), \\
& M \leq f(M+\varepsilon, m-\varepsilon) .
\end{aligned}
$$

These inequalities yields

$$
\begin{gathered}
m \geq \frac{a m^{3}+b m M^{2}+c m^{2} M+d M^{3}}{A m^{3}+B m M^{2}+C m^{2} M+D M^{3}}, \\
M \leq \frac{a M^{3}+b m^{2} M+c m M^{2}+d m^{3}}{A M^{3}+B m^{2} M+C m M^{2}+D m^{3}} .
\end{gathered}
$$

So,

$$
\begin{aligned}
& m M \geq \frac{M\left(a m^{3}+b m M^{2}+c m^{2} M+d M^{3}\right)}{A m^{3}+B m M^{2}+C m^{2} M+D M^{3}}, \\
& m M \leq \frac{m\left(a M^{3}+b m^{2} M+c m M^{2}+d m^{3}\right)}{A M^{3}+B m^{2} M+C m M^{2}+D m^{3}} .
\end{aligned}
$$

Hence

$$
\begin{gathered}
\frac{M\left(a m^{3}+b m M^{2}+c m^{2} M+d M^{3}\right)}{A m^{3}+B m M^{2}+C m^{2} M+D M^{3}} \\
-\frac{m\left(a M^{3}+b m^{2} M+c m M^{2}+d m^{3}\right)}{A M^{3}+B m^{2} M+C m M^{2}+D m^{3}} \leq 0
\end{gathered}
$$

which can be written

$$
(M-m) R(m, M)=0,
$$

where

$$
R(m, M)=\frac{L(m, M)}{K(m, M)},
$$

and

- $L(m, M)=d A\left(m^{6}+M^{6}\right)+p_{1} m M\left(m^{4}+M^{4}\right)+$ $p_{2} m^{2} M^{2}\left(m^{2}+M^{2}\right)+p_{3} m^{3} M^{3}$,

- $K(m, M)=\left(A m^{3}+B m M^{2}+C m^{2} M+D M^{3}\right)\left(A M^{3}+\right.$ $\left.B m M^{2}+C m^{2} M+D m^{3}\right)$.

Since

$$
R(m, M)>0
$$

we get

$$
M \leq m
$$

So, $m=M=\bar{x}$.

By the same arguments, we can easily prove the following theorem.

\section{Theorem 24 Let}

$$
\begin{aligned}
q_{1} & =(B+D) a+D c-A d, \\
q_{2} & =(B+C+D) a+(-A+D) b+(B+D) c-(A+C) d, \\
q_{3} & =(A+B+C+D) a+(-A+B-C+D) b \\
& +(-A+B+C+D) c+(-A-C-B+D) d .
\end{aligned}
$$

Assume that 
- $\frac{a}{A} \leq \min \left(\frac{b}{B}, \frac{c}{C}\right)$,

- $\frac{d}{D} \geq \max \left(\frac{b}{B}, \frac{c}{C}\right)$,

- $3 r_{3}+r_{4} \leq 0$,

- $\frac{-2\left(2 r_{1}+r_{2}+3 r_{3}+r_{4}+r_{5}+2 r_{6}\right)}{(a+b+c+d)(A+B+C+D)}<1$,

- $q_{1}, q_{2}, q_{3} \geq 0$.

Then the equilibrium point $\bar{x}=\frac{a+b+c+d}{A+B+C+D}$ of Eq.(1) is globally asymptotically stable.

Theorem 25 Assume that $q_{1}, q_{2}, q_{3} \geq 0$. Then Eq.(1) has no positive prime period two solution.

Proof. For the sake of contradiction, assume that there exist distinct positive real numbers $\alpha$ and $\beta$, such that

$$
\ldots, \alpha, \beta, \alpha, \beta, \ldots
$$

is a period two solution of Eq.(1). Then, $\alpha, \beta$ satisfy the system

$$
\alpha=f(\beta, \alpha), \beta=f(\alpha, \beta) .
$$

Thus, we have

$$
\beta f(\beta, \alpha)=\alpha f(\alpha, \beta)
$$

which gives

$$
(\beta-\alpha) S(\alpha, \beta)=0
$$

where

$$
S(\alpha, \beta)=\frac{F(\alpha, \beta)}{K(\alpha, \beta)}
$$

and

- $F(\alpha, \beta)=a D\left(\alpha^{6}+\beta^{6}\right)+q_{1} \alpha \beta\left(\alpha^{4}+\beta^{4}\right)+$ $q_{2} \alpha^{2} \beta^{2}\left(\alpha^{2}+\beta^{2}\right)+q_{3} \alpha^{3} \beta^{3}$

- $K(\alpha, \beta)=\left(A \beta^{3}+B \beta \alpha^{2}+C \beta^{2} \alpha+D \alpha^{3}\right)\left(A \alpha^{3}+\right.$ $\left.B \alpha \beta^{2}+C \alpha^{2} \beta+D \beta^{3}\right)$.

Since

$$
S(\alpha, \beta)>0 .
$$

We get $\beta=\alpha$, which is a contradiction.

\section{Numerical examples}

In order to illustrate and support our theoretical discussions, we consider interesting numerical examples in this section.

\section{Example 31 Let}

$(a, b, c, d, A, B, C, D)=\left(1, \frac{3}{2}, 1, \frac{1}{2}, 1,2,2, \frac{3}{2}\right) \quad$ and $\left(x_{-1}, x_{0}\right)=(0.5,0.7)$. Then all conditions of theorem (23) are satisfied and we have the following results:

\begin{tabular}{|l|l||l|l|}
\hline$n$ & $\left|x_{n}-\bar{x}\right|$ & $n$ & $\left|x_{n}-\bar{x}\right|$ \\
\hline 1 & 0.0507956108 & 31 & 0.00 \\
2 & 0.0072974127 & 32 & $0.1 .10^{-9}$ \\
3 & 0.0134001561 & 33 & $0.1 .10^{-9}$ \\
4 & 0.0014909700 & 34 & $0.2 .10^{-9}$ \\
\hline \hline$n$ & $\left|x_{n}-\bar{x}\right|$ & $n$ & $\left|x_{n}-\bar{x}\right|$ \\
\hline 61 & $0.3 .10^{-9}$ & 91 & $0.2 .10^{-9}$ \\
62 & $0.2 .10^{-9}$ & 92 & 0.00 \\
63 & $0.2 .10^{-9}$ & 93 & $0.3 .10^{-9}$ \\
64 & 0.00 & 94 & $0.2 .10^{-9}$ \\
\hline
\end{tabular}

Example 32 Let

$(a, b, c, d, A, B, C, D)=\left(1,2,2, \frac{3}{2}, 1, \frac{3}{2}, 1, \frac{1}{2}\right) \quad$ and $\left(x_{-1}, x_{0}\right)=(1.5,2.7)$. Then all conditions of theorem (24) are satisfied and we have the following results:

\begin{tabular}{|l|l||l|l|}
\hline$n$ & $\left|x_{n}-\bar{x}\right|$ & $n$ & $\left|x_{n}-\bar{x}\right|$ \\
\hline 1 & 0.206160365 & 31 & $0.251 .10^{-6}$ \\
2 & 0.275199172 & 32 & $0.158 .10^{-6}$ \\
3 & 0.108454901 & 33 & $0.99 .10^{-7}$ \\
4 & 0.091283226 & 34 & $0.62 .10^{-7}$ \\
\hline \hline$n$ & $\left|x_{n}-\bar{x}\right|$ & $n$ & $\left|x_{n}-\bar{x}\right|$ \\
\hline 61 & 0.00 & 91 & 0.00 \\
62 & 0.00 & 92 & 0.00 \\
63 & $0.1 .10^{-8}$ & 93 & $0.1 .10^{-8}$ \\
64 & 0.00 & 94 & 0.00 \\
\hline
\end{tabular}

\section{References}

[1] C. Cinar, On the positive solutions of the difference equation system $x_{n+1}=1 / y_{n}, y_{n+1}=y_{n} / x_{n-1} y_{n-1}$, Appl. Math. Comput., 158, 303-305 (2004).

[2] C. W. Clark, A delayed recruitment of a population dynamics with an application to baleen whale populations, J. Math. Biol., 3, 381-391 (1976).

[3] E. M. Elabbasy and E. M. Elsayed, On the global attractivity of difference equation of higher order, Carpathian J. Math., 24, 45-53 (2008).

[4] E. M. Elsayed, On the solution of some difference equations, European J. Pure Appl. Math., 4, 287-303 (2011).

[5] E. M. Elsayed, On the dynamics of a higher order rational recursive sequence, Commun. Math. Anal., 12, 117-133 (2012).

[6] B. D. Iricănin and N. Touafek, On a second order max-type system of difference equations, Indian J. Math., 54, 119-142 (2012).

[7] V. L. Kocic and G. Ladas, Global bahavior of nonlinear difference equations of higher order with applications, Kluwer Academic Publishers, Dordrecht, (1993).

[8] M. R. S. Kulenovic and G. Ladas, Dynamics of second order rational difference equations with open problems and conjectures, Chapman and Hall, CRC Press, (2001).

[9] A. S. Kurbanli , C. Cinar and I. Yalçinkaya, On the behavior of positive solutions of the system of rational difference equations, Math. Comput. Modelling, 53, 12611267 (2011), 
[10] I. Ozturc, F. Bozkurt and S. Ozen, On the difference equation $y_{n+1}=\frac{\alpha+\beta e^{-y_{n}}}{\gamma+y_{n-1}}$, Appl. Math. Comput., 181, 13871393 (2006).

[11] I. Yalçinkaya, On the difference equation $x_{n+1}=\alpha+\frac{x_{n-2}}{x_{n}^{k}}$, Fasc. Math., 42, 133-139 (2009). 